\title{
Implante de peso de ouro: complicações precoces e tardias
}

\author{
Gold weight implantation:premature and latecomplications
}

\author{
Tânia Pereira Nunes ${ }^{1}$ \\ Mariluze Sardinha ${ }^{2}$ \\ Ivana Cardoso Pereira ${ }^{3}$ \\ Patrícia Lunardelli ${ }^{4}$ \\ Suzana Matayoshi ${ }^{5}$
}

\begin{tabular}{|l|}
\hline \multicolumn{1}{|c|}{ RESUMO } \\
\hline Objetivos: Avaliar as indicações, os resultados e as complicações obser- \\
vadas nos pacientes submetidos ao implante de peso de ouro para \\
correção do lagoftalmo paralítico. Métodos: Vinte prontuários de pacientes \\
com lagoftalmo secundário à paralisia facial de diversas etiologias, que \\
foram submetidos à colocação do implante de ouro na pálpebra superior \\
do lado afetado, foram examinados retrospectivamente. Resultados: A \\
causa mais frequiente de lagoftalmo paralítico foi pós-cirurgia de neurinoma \\
do acústico (40\%). Complicações precoces e tardias ocorreram em $40 \%$ dos \\
implantes colocados. Quatro pacientes (20\%) apresentaram reação inflama- \\
tória local nos primeiros meses de pós-operatório. Dois pacientes (10\%) \\
apresentaram afinamento da pele e do músculo orbicular sobre o peso de \\
ouro, após 4 e 7 anos do implante, respectivamente. Um paciente (5\%) \\
apresentou deslocamento do peso de ouro após 3 anos de sua colocação \\
e outro paciente (5\%), extrusão do peso de ouro tardiamente, após 10 anos \\
do implante. Conclusões: Nesta série, foi alto oíndice de complicações com \\
o implante de ouro (40\%). As complicações foram divididas em precoces, \\
possivelmente relacionadas à impureza do material, e complicações tardias, \\
devido à evolução do quadro da paralisia facial que apresenta diminuição \\
do tônus muscular.
\end{tabular}

Descritores: Ouro; Implante de prótese; Paralisia facial; Doenças palpebrais; Complicações pós-operatórias; Humano; Masculino; Feminino; Adulto; Meia-idade
Trabalho realizado no Setor de Plástica Ocular, da Clínica Oftalmológica do Hospital das Clínicas da USP - São Paulo (SP).

${ }^{1}$ Médica pós-graduanda da Clínica Oftalmológica da Faculdade de Medicina da Universidade de São Paulo - USP - São Paulo (SP) - Brasil.

${ }^{2}$ Médica pós-graduanda da Clínica Oftalmológica da Faculdade de Medicina da USP - Ribeirão Preto (SP) Brasil.

${ }_{3}^{3}$ Médica estagiária do Setor de Plástica Ocular da Clínica Oftalmológica da Faculdade de Medicina da USP - São Paulo (SP) - Brasil.

${ }^{4}$ Médica estagiária do Setor de Plástica Ocular da Clínica Oftalmológica da Faculdade de Medicina da USP São Paulo (SP) - Brasil.

${ }^{5}$ Chefe do Setor de Plástica Ocular da Faculdade de Medicina da USP - São Paulo (SP) - Brasil.

Endereço para correspondência: Tânia Pereira Nunes Rua Cristiano Viana,116 - Apto. 32 - São Paulo (SP) CEP 05411-000

E-mail: nunes32@bol.com.br

Recebido para publicação em 13.12.2005

Última versão recebida em 24.03.2007

Aprovação em 07.05.2007

Nota Editorial: Depois de concluída a análise do artigo sob sigilo editorial e com a anuência da Dra. Simone H.D. Von Faber Bison sobre a divulgação de seu nome como revisora, agradecemos sua participação neste processo.

\section{INTRODUÇÃO}

O lagoftalmo paralítico (Figura 1), que se caracteriza pela incapacidade de fechamento completo das pálpebras resultante da paralisia facial, pode levar à exposição corneal, causando desde leve desconforto ocular até casos extremos, como a perfuração ocular. Por ser esta uma situação potencialmente grave, é importante que o oftalmologista esteja preparado para indicar o melhor tratamento diante de um caso de paralisia facial ${ }^{(1)}$.

O tratamento do lagoftalmo paralítico pode ser clínico, que consiste basicamente no uso de medicamentos lubrificantes, associado ou não ao cirúrgico $^{(1)}$. Uma técnica cirúrgica simples é a tarsorrafia, que pode ser temporal, medial ou ambos ${ }^{(2)}$; apesar de ser um procedimento de fácil execução, apresenta como desvantagens pobre resultado estético e redução do campo visual periférico. Outra técnica cirúrgica bastante utilizada é o implante de peso de ouro. O peso de ouro colocado na pálpebra superior do lado afetado proporciona a oclusão palpebral pela ação da gravidade com o relaxamento do músculo levantador da pálpebra superior (MLPS). É considerado um método fisiológico, pois não interfere na estética facial, na abertura palpebral e preserva o campo de visão. Outra vantagem é ser um 
método reversível, podendo ser removido sem alterar a anatomia palpebral ${ }^{(1-2)}$.

Este estudo foi feito com o objetivo de avaliar as indicações, bem como os resultados e as complicações observadas nos pacientes submetidos ao implante de peso de ouro para correção do lagoftalmo paralítico.

\section{MÉTODOS}

Foram examinados retrospectivamente 20 prontuários de pacientes com lagoftalmo secundário à paralisia facial de diversas etiologias, que foram submetidos à colocação de peso de ouro na pálpebra superior do lado afetado, acompanhados no Setor de Plástica Ocular da clínica oftalmológica do Hospital das Clínicas - FMUSP, São Paulo.

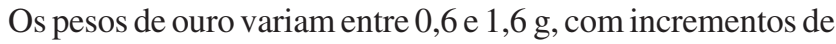
$0,2 \mathrm{em} 0,2 \mathrm{~g}^{(2-3)}$. A escolha do peso apropriado foi feita fixando-os na pálpebra superior do paciente com fita adesiva (Figura 2): o peso ideal produziu o fechamento palpebral completo sem causar ptose. A maioria dos pesos utilizada neste trabalho foi de $1,2 \mathrm{~g}$.

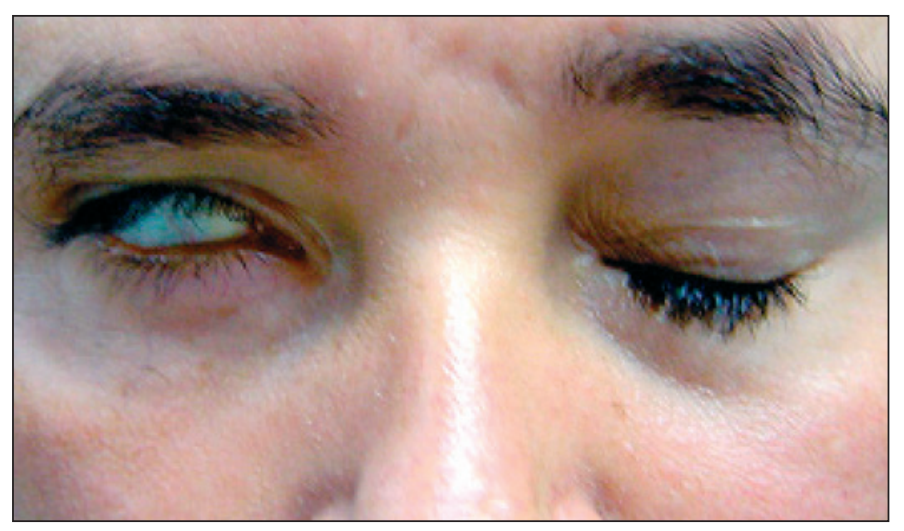

Figura 1 - Lagoftalmo paralítico

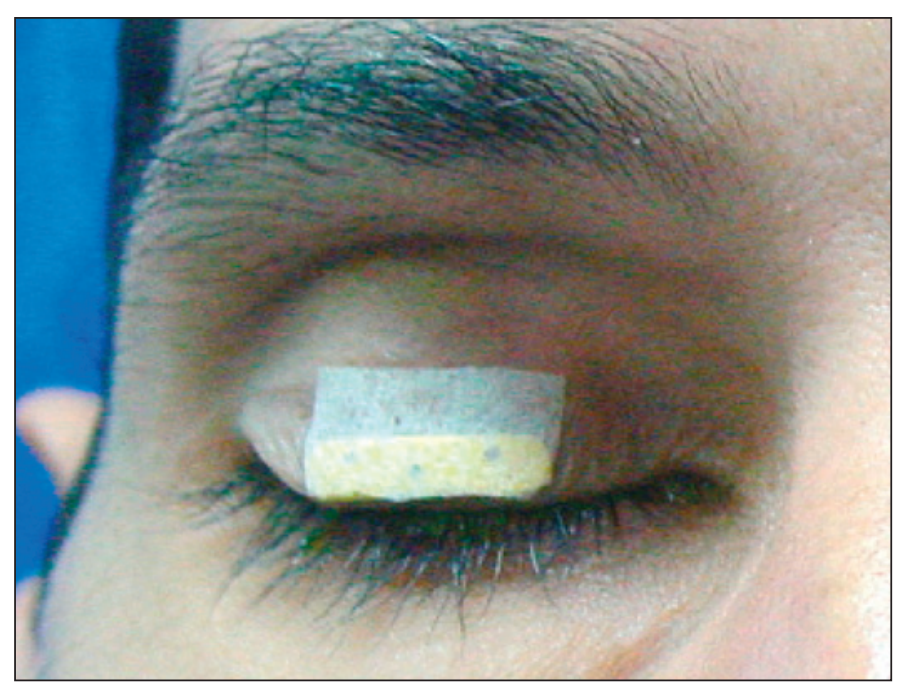

Figura 2 - Teste para escolha do peso ideal
O implante do peso de ouro foi realizado sob anestesia local em todos os casos. Após infiltração de lidocaína a $2 \%$ com vasoconstritor na pálpebra superior do lado paralisado, fez-se incisão horizontal, entre 10 e 15 mm, com bisturi lâmina 15, na altura do sulco palpebral. Realizou-se dissecção do músculo orbicular expondo a placa tarsal. O peso de ouro (Figura 3) foi fixado sobre o tarso com três pontos, sendo o furo central colocado na direção de uma linha vertical imaginária, traçada do centro da pupila ao tarso e os outros dois pontos fixados medial e lateral a este primeiro ponto, utilizando-se o fio de seda 6.0. Realizou-se sutura do plano muscular sobre o peso de ouro, com fio absorvível 6.0 com o objetivo de proteger o peso de ouro e prevenir possível deslocamento ou extrusão. A sutura da pele foi realizada com seda ou mononylon $6.0^{(2)}$.

O período de seguimento variou entre 2 meses e 10 anos.

\section{RESULTADOS}

Foram colocados 20 implantes de ouro em 20 pacientes, sendo 9 pacientes (45\%) do sexo masculino e $11(55 \%)$, do feminino. A idade variou entre 16 e 86 anos (média: 51 anos).

As causas das paralisias faciais estão listadas no quadro 1.

Dentre os 20 pesos de ouro colocados, ocorreram complicações pós-operatórias em $40 \%$ dos casos. As complicações foram divididas em precoces e tardias. Quatro pacientes (20\%)

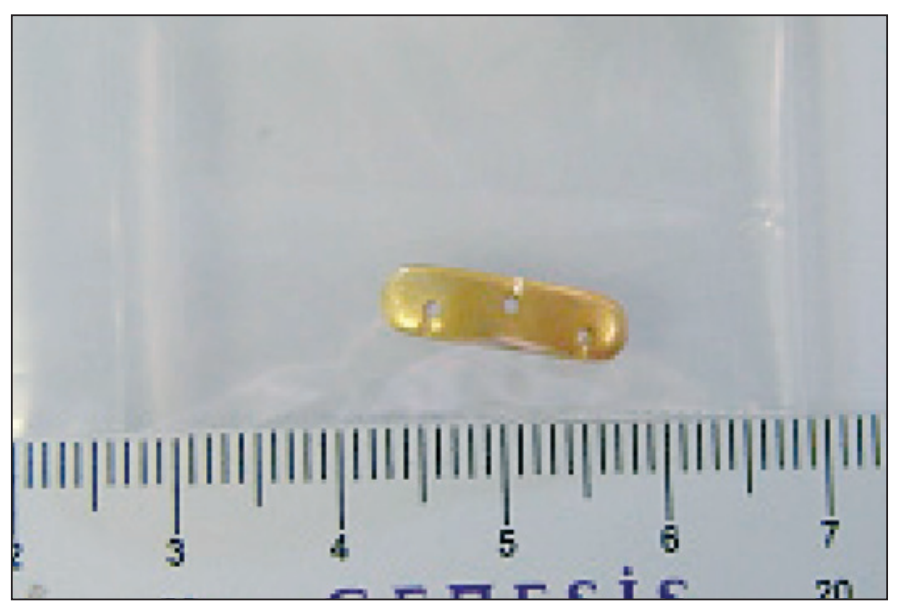

Figura 3 - Peso de ouro

\section{Quadro 1. Paralisia facial: diversas etiologias}

\begin{tabular}{lcr} 
Etiologias & No de pacientes & $\%$ \\
Congênita & 1 & 5 \\
Pós-remoção do neurinoma do acústico & 8 & 40 \\
Neurinoma do acústico & 1 & 5 \\
Outro tumor do ângulo ponto-cerebelar & 2 & 10 \\
Acidente vascular cerebral & 1 & 5 \\
Herpes zoster & 2 & 10 \\
Ferimento por arma de fogo & 2 & 10 \\
Etiologia desconhecida & 3 & 15 \\
\hline
\end{tabular}


apresentaram reação inflamatória local nos primeiros meses de pós-operatório, sendo necessário a retirada do peso de ouro.

Dois pacientes (10\%) apresentaram afinamento da pele e do músculo orbicular sobre o peso de ouro (Figura 4). Os pacientes foram submetidos ao implante de peso de ouro há 4 anos e 7 anos, respectivamente.

Em um paciente (5\%), que apresentou deslocamento do peso de ouro após 3 anos da sua colocação, a sua remoção cirúrgica fez-se necessária. Outro paciente (5\%) apresentou extrusão do peso de ouro tardiamente, após 10 anos do implante.

\section{DISCUSSÃO}

As causas de paralisia facial podem ser: idiopática (também conhecida por paralisia facial de Bell), infecciosa, traumática e tumoral ${ }^{(2-4)}$. Em nosso estudo, a causa mais freqüente ( $40 \%)$ foi pós-cirurgia de neurinoma do acústico. Esta causa também prevaleceu em outro estudo e seus autores acreditam que, por ser um quadro geralmente irreversível, o tratamento cirúrgico das alterações palpebrais é mais freqüente que em outras causas de paralisia, por exemplo, a paralisia de Bell ${ }^{(5)}$.

Várias técnicas cirúrgicas são utilizadas para o tratamento do lagoftalmo paralítico com diferentes graus de sucesso. O implante de peso de ouro tornou-se bastante popular pelas seguintes vantagens: procedimento rápido e simples, ser realizado sob anestesia local e bom resultado estético e funcional ${ }^{(6-7)}$.

Algumas complicações descritas do implante do peso de ouro são: blefaroptose no pós-operatório, implante visível esteticamente inaceitável, infecção, deslocamento ou extrusão do peso de ouro ${ }^{(3-4,6-8)}$.

Em quatro pacientes deste trabalho, observou-se reação tecidual que indicava o envolvimento de processo imunológico de rejeição. Foi necessária a retirada do implante, porém em quadro inflamatório leve, pode-se utilizar corticóide, tópico ou oral ${ }^{(9)}$. A impureza do ouro é uma possibilidade para

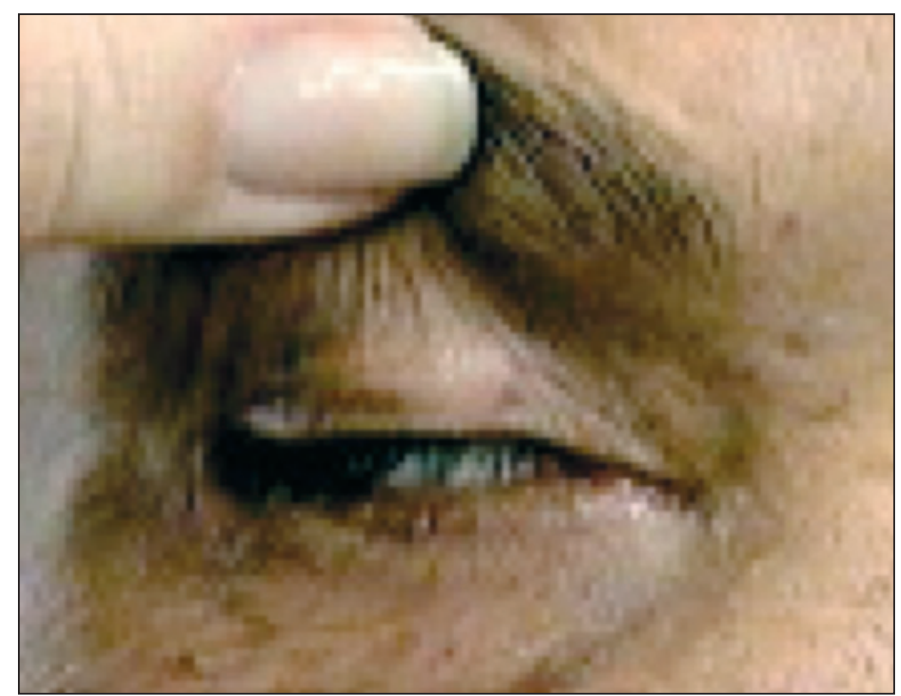

Figura 4 - Afinamento da pele e do músculo orbicular sobre o peso de ouro explicar a reação inflamatória local, e, portanto, o peso deve ser fabricado com ouro puro $(99,99 \%)$. Outra possibilidade é uma reação alérgica ao metal, apesar dos pacientes não terem historia prévia de alergia ao ouro. Um aspecto importante a ser considerado é saber a procedência do implante para certificar a pureza do ouro utilizado.

O afinamento da lamela anterior das pálpebras, pele e músculo orbicular, pode aparecer em quadros de paralisia facial de longa duração e facilitar o deslocamento ou a extrusão do peso de ouro. Dois pacientes, em nosso estudo, apresentaram afinamento da pele e do músculo orbicular sobre o peso de ouro. O seguimento pós-operatório era de 4 anos e 7 anos. Outras complicações que tivemos e que acreditamos estarem relacionadas com a diminuição do tônus da pele e do músculo orbicular são: deslocamento do peso de ouro após 3 anos da sua colocação e extrusão do peso de ouro, após 10 anos do implante. Algumas modificações no procedimento cirúrgico do implante de peso de ouro foram descritas para evitar tais complicações, como por exemplo, o uso de materiais como a fáscia lata, a fáscia temporal ou o pericárdio humano para recobrir o peso ou sua fixação na aponeurose do MLPS, sob o septo orbital $^{(6,8,10-11)}$.

\section{CONCLUSÕES}

Embora o implante de ouro seja um procedimento muito aceito em nosso meio, as complicações ocorrem em número razoável; as precoces estão relacionadas à impureza do material ou reação alérgica ao ouro, e as tardias, ao afinamento da lamela anterior da pálpebra.

\section{ABSTRACT}

Purpose: To evaluate the indications, the results and the complications seen in the patients submitted to gold weight implantation to correct paralytic lagophthalmos. Methods: Charts of 20 patients with lagophthalmos secondary to facial nerve palsy of diverse etiologies, which were submitted to gold weight implantation in the upper eyelid of the affected side, were retrospectively examined. Results: The most frequent cause of paralytic lagophthalmos was acoustic neurinoma after surgery (40\%). Early and late complications occurred in $40 \%$ of the implants. Four patients $(20 \%)$ presented a local inflammatory reaction in the first 3 months after surgery. Two patients $(10 \%)$ presented skin and orbicular muscle thinness over the gold weight 4 and 7 years after the implant, respectively. One patient $(5 \%)$ presented gold weight displacement after 3 years and another patient (5\%) had late gold weight extrusion after 10 years. Conclusions: In this series, the complication rate of gold weight implantation was high (40\%). The complications were divided into early, possibly related to the material impurity and the late, due to the evolution of the facial nerve palsy that presented a decrease in muscle tonus. 
Keywords: Gold; Prosthesis and implants; Facial paralysis; Eyelid diseases; Postoperative complications; Human; Male; Female; Adult; Middle aged

\section{REFERÊNCIAS}

1. Portellinha WM, Genta CB, Sobrinho EFA, Cunha MC, Moura EM. Paralisia facial - lagoftalmo. In: Soares EJ, Moura EM, Gonçalves JO. Cirurgia Plástica Ocular. São Paulo: Roca; 1997. p.193-202.

2. Forno EA. Lagoftalmo paralítico. In: Matayoshi S, Forno EA, Moura EM. Manual de Cirurgia Plástica Ocular. São Paulo: Roca; 2004. p.79-86.

3. Seiff SR, Sullivan JH, Freeman LN, Ahn J. Pretarsal fixation of gold weights in facial nerve palsy. Ophthal Plast Reconstr Surg. 1989;5(2):104-9.

4. Harrisberg BP, Singh RP, Croxson GR, Taylor RF, McCluskey. Long-term outcome of gold eyelid weights in patients with facial nerve palsy. Otol Neurotol. 2001;22(3):397-400.
5. Linhares JD, França VP, Soares, EJC. Lagoftalmo paralítico: uma proposta de classificação clínico-cirúrgica. Arq Bras Oftalmol. 1995;58(5):319-24.

6. Tower RN, Dailey RA. Gold weight implantation: a better way? Ophthal Plast Reconstr Surg. 2004;20(3):202-6. Comment in: Ophthal Plast Reconstr Surg. 2005;21(1):80; author reply 80 .

7. Foda HM. Surgical management of lagophthalmos in patients with facial palsy. Am J Otolaryngol. 1999;20(6):391-5.

8. Gladstone GJ, Nesi FA. Management of paralytic lagophthalmos with a modified gold-weight implantation technique. Ophthal Plast Reconstr Surg. 1996;12(1):38-44.

9. Dinces EA, Mauriello JA Jr, Kwartler JA, Franklin M. Complications of gold weight eyelid implants for treatment of fifth and seventh nerve paralysis. Laryngoscope. 1997;107(12):1617-22.

10. Jobe RP. A technique for lid loading in the management of the lagophthalmos of facial palsy. Plast Reconstr Surg. 1974;53(1):29-32.

11. Foster JA, Perry JD, Cahill KV, Holck DE, Kugler L. Processed human pericardium barrier for gold weight implantation. Ophthal Plast Reconstr Surg. 2004;20(2):107-9.

12. Thomas DA, Khalifa YM. Temporalis fascia in the management of gold eyelid weight extrusion. Ophthal Plast Reconstr Surg. 2005;21(2):153-5. 